\title{
LA BIBLIOTECA ES SERVICIO (Y EN ELLO ESTÁ NUESTRO FUTURO)
}

\author{
A library is a service (and our future depends on it)
}

José-Pablo Gallo-León

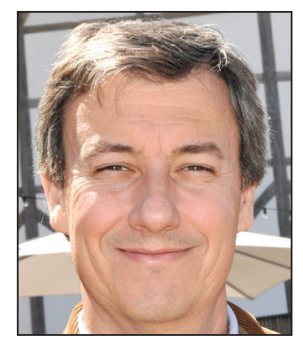

José-Pablo Gallo-León es doctor en comunicación y documentación por la Universidad de Murcia, licenciado en geografía e historia (arte) y magíster en biblioteconomía por la Universidad Complutense de Madrid. Ha sido director de la Biblioteca Regional de Murcia y de la Biblioteca de la Universidad Miguel Hernández. Su campo de investigación se orienta hacia los servicios en las bibliotecas, su futuro y la arquitectura bibliotecaria. Ha participado y coordinado grupos de trabajo de Rebiun y del Consejo de Cooperación Bibliotecaria (CCB), siendo el más reciente el Grupo estratégico para el estudio de prospectiva sobre la biblioteca. http://orcid.org/0000-0002-8236-4275

Universidad de Alicante. Biblioteca de Educación Carretera de San Vicente del Raspeig, s/n. 03690 San Vicente del Raspeig (Alicante), España jpablo.gallo@ua.es

\section{Resumen}

A pesar de la imagen que la sociedad tiene de ellas, las bibliotecas siempre se han fundamentado más en el servicio prestado que en su colección, que es una herramienta para el mismo. Por ello los servicios bibliotecarios deben ser la base para su desarrollo y supervivencia. Su diseño debe partir de las necesidades de los usuarios, pues ellos son su razón de ser. De hecho, toda la biblioteca se debe orientar hacia los usuarios, intentando ofertar unos servicios que cubran la necesidades de una población objetivo lo más amplia posible. Las tecnologías que han supuesto un cambio disruptivo en el modelo de biblioteca nos pueden ser al mismo tiempo de gran ayuda para este objetivo.

\section{Palabras clave}

Servicios bibliotecarios; Bibliotecas; Prospectiva; Futuro; Usuarios de la biblioteca.

\begin{abstract}
Despite their public image, libraries have always been based more on the services provided than on the collection, which is only a tool for the service. Therefore, library services should be the basis for libraries' development and survival. The design of those services must be based on the needs of their users, who are the reason why libraries exist. As a matter of fact, the entire library should be addressed to the users, offering services that cover the needs of the broadest possible target population. Technologies that have brought about disruptive change in the model of the library can be also helpful to this purpose.
\end{abstract}

\section{Keywords}

Library services; Users' services; Libraries; Future; Library patrons.

Gallo-León, José-Pablo (2015). "La biblioteca es servicio (y en ello está nuestro futuro)”. El profesional de la información, v. 24, n. 2, pp. 87-93.

http://dx.doi.org/10.3145/epi.2015.mar.01

\section{Introducción: la biblioteca es servicio}

"Nunca ha existido un tiempo más estimulante para dedicarse a las bibliotecas: es a la vez amenazador y sugerente"

Hablar de servicios bibliotecarios es hablar de biblioteca. Al contrario de lo que su propio nombre indica, la biblioteca no es una colección, sino un servicio. De lo contrario nada la diferenciaría de un almacén. Así ha sido desde el principio: las bibliotecas aparecieron para asegurar el acceso a los libros, independientemente de su formato (códice, rollo, tableta...). La preservación sólo era una parte de esa misión. Por tanto, su función primera fue la de servicio de información, de herramienta de acceso a la información para ayudar al conocimiento. 


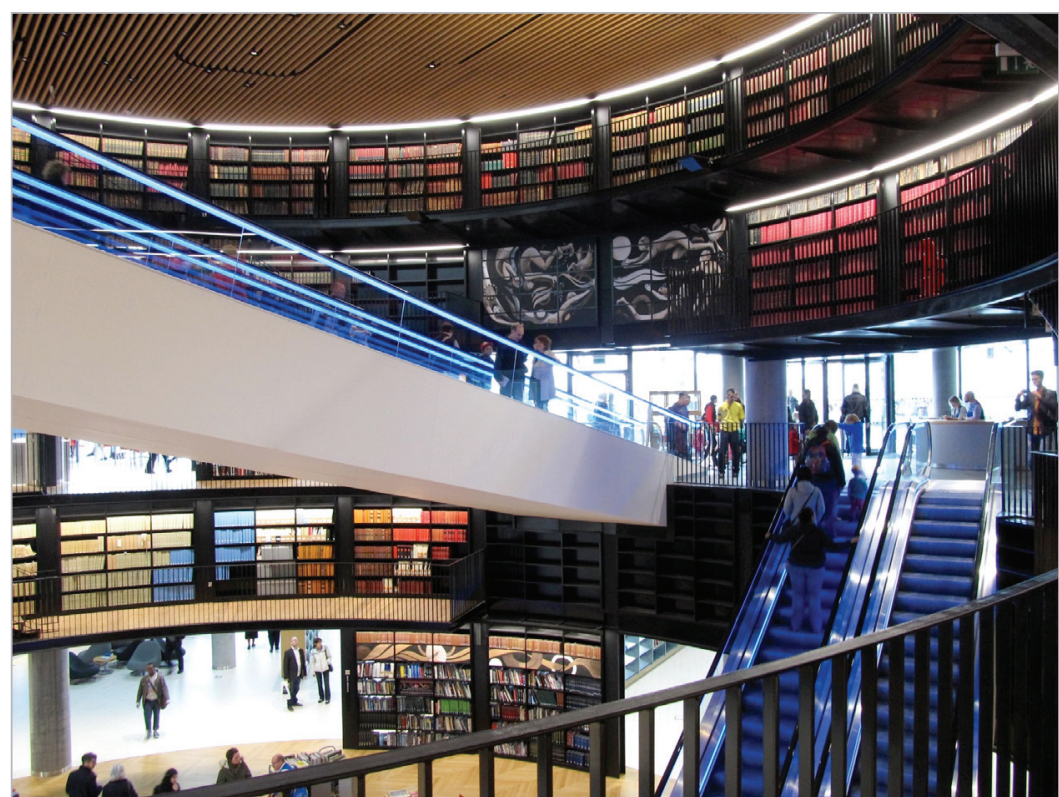

Figura 1. La nueva (2013) Library of Birmingham: el libro, aún protagonista, aunque como objeto simbólico de decoración. Fuente: jo-marshall (Flickr: Levels) [CC BY 2.0], vía Wikimedia Commons

http://commons.wikimedia.org/wiki/File\%3ALibraryOfBirmingham-Levels.jpg

De esta primitiva función de servicio han surgido las demás, vinculadas a ella y como consecuencia de la misma. Un ejemplo es el servicio de referencia ${ }^{2}$, resultado lógico de que la información se organizase y localizase en la biblioteca. Sucede igual con el resto de servicios, que han ido transformando el concepto de biblioteca en "una organización prestadora de servicios" (Lozano-Díaz, 2008).

La biblioteca es un servicio, no una colección de libros. Si no, nada la diferenciaría de un almacén

Sin embargo, el servicio primario de la biblioteca como organizadora/facilitadora de la información se encuentra en crisis. Los usuarios tienen otras vías de conseguir la información o no nos identifican con ellas. La gestión de la colección, que ha sido el eje de nuestro trabajo como bibliotecarios, pierde aparentemente su necesidad. Múltiples voces hablan de la inminente desaparición de la biblioteca, si bien es cierto que esto sucede desde hace más de 40 años.

¿Cómo no vamos a estar alarmados si hay estudios que indican que sólo el $1 \%$ de los estudiantes empiezan sus búsquedas de información por el catálogo? Evidentemente tenemos un grave problema (Choy, 2011, p. 63). Con las nuevas formas de investigación hemos dejado de ser la fuente primaria, aunque quizá en España nunca lo hayamos llegado a ser de forma clara, sustituyéndonos por el entorno de colegas. Las tecnologías de la información y la comunicación han traído cambios profundos que han sido anunciados repetidamente y que, como señala Anglada (2012), no son cosméticos ni acumulativos, sino estructurales y disruptivos.

Todo ello hace que los bibliotecarios nos preguntemos por nuestro futuro; y sobre si tenemos algún futuro. Así se evidencia en la avalancha de estudios y artículos, existiendo in- cluso un Center for the Future of Libraries fundado por la $A L A$ en $2014^{3}$. Tenemos una opción clara frente al pesimismo: si la biblioteca es servicio, lo que debemos hacer es potenciarlos y desarrollar otros nuevos para adaptarnos a las circunstancias. Una transformación que puede implicar cambios radicales, incluso de nuestra propia denominación. De eso se va a hablar en este texto: de los servicios como nuestro futuro y del desarrollo de los mismos. Sin hacer una recopilación de los más novedosos, que se puede encontrar en otros lugares ${ }^{4}$, sino reflexionando sobre su concepción, diseño e implementación.

\section{Servicios que se adaptan a la realidad: asegurando nuestro futuro}

Dicho lo anterior, y viendo los resultados de la Summit on the Future of Libraries de la ALA (Bolt, 2014) y algunos trabajos más sobre el futuro de las bibliotecas, podría parecer más oportuno titular este trabajo "Las bibliotecas ya no serán bibliotecas nunca más". Aunque quizá exagerado, puede dar una idea aproximada de la radicalidad de los cambios en los servicios que se están produciendo y deben realizarse. Al menos así debe parecer desde el punto de vista de un observador externo.

Cualquier bibliotecario sabe por experiencia que la sociedad en general asocia todavía el concepto biblioteca con el libro impreso, incrementándose incluso esa percepción a pesar de los rápidos cambios de los últimos años. En Estados Unidos el porcentaje de personas que así lo creían pasó del 69\% en 2005 al 75\% en 2010 (Anglada, 2014, p. 607) ( $^{5}$. Mejor no imaginar lo que ocurre en España. Por ello debemos realizar un gran esfuerzo por cambiar la idea común de biblioteca que tiene la sociedad, para aproximarla a ese entorno de servicios para el intercambio y la generación de conocimiento que pretendemos. Esta biblioteca orientada al futuro se debe distinguir y valorar más por el alcance y calidad de sus servicios que por la riqueza de sus colecciones, como indica Walker (2011) que sucedía hasta ahora.

Los bibliotecarios suelen expresar bastante optimismo sobre la situación, vista su demostrada capacidad evolutiva. Por ejemplo, en la citada Summit de la ALA los participantes reaccionaron en este sentido frente a los acicates de los ponentes, que expresaban mayores dudas. Ciertamente, las bibliotecas "han sabido hacer evolucionar los servicios tradicionales a la vez que, cabalgando las tecnologías, han creado nuevos servicios siguiendo la estela de las necesidades en parte tradicionales, en parte cambiantes, de sus usuarios" (Anglada, 2012). Todo ello bajo la fuerte presión de un cambio tecnológico radical.

Debemos proseguir al menos con la evolución y adaptación continua al ritmo que cambian las necesidades de los usuarios. Y decimos "al menos" porque quizá no baste con esta evolución. A veces los cambios serán también radicales, y para ello hace falta una gran visión y valentía en la gestión. 
Se necesitan tanto ideas frescas como la constante reevaluación de las viejas ideas (Casey; Savastinuk, 2006). Esto significa la innovación como factor de supervivencia (Lozano-Díaz, 2008), más la revisión continua de conceptos. Pero también podemos usar el conocimiento grupal: adaptar ejemplos de éxito usando la red de conocimiento tejida durante años por los bibliotecarios, aunque con remiendos por la falta de visión, absurda competitividad y egoísmo, afortunadamente muy minoritarios. A modo de los colegios invisibles de Crane, los bibliotecarios continúan compartiendo información de interés común (Gallo-León, 2008).

Si nos preguntamos entonces hacia dónde orientaremos nuestros servicios, hay que tener claro que la situación es cambiante, por lo que la prioridad es determinar cuáles son las principales necesidades de nuestros usuarios. Las bibliotecas tendrán que valorar qué tipos de servicios van a ofrecer de acuerdo con la evolución del entorno, combinando en la oferta presenciales y remotos con la doble dimensión física y digital. Desde esta perspectiva híbrida y en constante renovación, se ofertan servicios de ambas clases que se complementan entre sí, mientras que cada usuario elige la modalidad en función de sus necesidades.

Tal como indica el estudio Prospectiva 2020 (Gallo-León, 2013), la generación de nuevos servicios físicos y digitales se encaminará hacia la potenciación de la biblioteca como instrumento para crear comunidades, planteando un espacio físico y virtual que funcione como punto de encuentro o ágora. En todos estos casos, la potencia como instrumento educativo formal e informal de la biblioteca a lo largo de la vida aparece como constante y fundamento.

En un contexto de revolución digital las bibliotecas se replantean cómo gestionar la información: ampliando los soportes en los que se encuentra, trabajando en red, asegurando a los usuarios el acceso a la información o generando nuevos servicios. Buscan así alejarse de la llamada amenaza Coffman (Coffman, 2012) ${ }^{6}$, por la que proveedores de contenidos digitales pueden ofrecer a la institución y usuarios un servicio similar al de la biblioteca, pero ampliado y más barato.

Además de estos servicios vinculados a nuestras funciones tradicionales, la biblioteca se configurará como espacio social de desarrollo del conocimiento, ofreciendo herramientas y actividades de manera personalizada o colectiva. Las bibliotecas, como dice Frey, están pasando de ser sitios para consumir cosas a sitios donde hacer cosas (Thomas Frey, cit. por Bolt, 2014). Y es que se nos plantea una gran variedad de oportunidades para el desarrollo de nuevas propuestas, con temas como las nuevas formas de investigación, servicios para adolescentes, aprovechamiento de los big data, movilidad, humanidades digitales, servicios a personas con

discapacidad, interculturales, información de proximidad, internet de las cosas, etc.

Las posibilidades son amplísimas, abiertas a nuestra capacidad de innovación, hasta conseguir ofrecer servicios que sean valorados y usados. Unido a esto, nuestro espacio físico y virtual debe ser el sitio en el que apetezca estar, al que se quiera ir. Una de las claves es conseguir ese tan nombrado tercer lugar de Ray Oldenburg ${ }^{7}$, pero sin servicios que lo sostengan quedaría en nada. Son los servicios los que lo transforman en un ágora, en una plaza o punto de encuentro e intercambio.

\section{Debido a los cambios, puede parecer que las bibliotecas ya no serán bibliote- cas nunca más}

Estos servicios deben responder a los principios tradicionales de las bibliotecas: acceso igualitario, equidad, neutralidad, respeto de la privacidad, voluntad de servicio, etc. Junto a ellos se adoptan nuevos valores, íntimamente relacionados con los anteriores, como la sostenibilidad o la responsabilidad social corporativa. Con el actual desarrollo de las redes sociales y la utilización de aplicaciones externas ${ }^{8}$, se hace más importante que nunca el equilibrio entre privacidad personal, difusión y seguridad. Igualmente, no podemos perder valores como la universalidad, la "biblioteca para todos", tratando de alcanzar un sector de público al que no llegamos, como nos recordaba el Grupo Durga (2012) con relación a una biblioteca orientada a los adolescentes en París.

Para todo ello se necesita aplicar una gestión flexible, valiente y con visión; así como una apuesta clara y decidida por la cooperación. Más aún en un entorno de restriccio- 
nes presupuestarias continuadas. Igualmente aparece como necesario que los bibliotecarios tengan nuevas capacidades y conocimientos, perfiles flexibles y en constante cambio. A pesar de la adaptabilidad demostrada hasta el momento, los profesionales son señalados como una posible fuerza reaccionaria en contra de estos cambios por los propios bibliotecarios (Bolt, 2014). Se necesita un cambio de mentalidad, e incluso quizá de nombre, como señala Anglada (2014), pues la denominación bibliotecario aparece ligada al edificio y al libro en el imaginario colectivo y hasta en su etimología.

Relacionado con esto, como ejemplo, las Idea stores suprimieron el nombre de biblioteca porque o bien era desconocido por su mayoritario público inmigrante o bien tenía una connotación negativa (Berndtson, 2012). Personalmente me parece cuestionable la oportunidad de cambiar el nombre. Ya se ha intentado repetidamente con dudoso éxito antes [mediateca; sibid (servicio de información bibliográfica y documental); learning centre; e incluso crai, aunque estos modelos quieran implicar más cosas). Pero lo que sí es notable es la oportunidad de nombrar bien nuestro centro, proporcionando un alias atractivo. Por ejemplo, la Helsinky Central Library se llama Library 10 por estar situada en la antigua oficina postal del código 10. También las Idea stores son ejemplos de denominaciones atractivas.

\section{La biblioteca se debe distinguir más por el alcance y calidad de sus servicios que por la riqueza de sus colecciones}

Continuando con el argumento sobre el personal, indudablemente se necesitan nuevas facultades $y$, al tiempo, dar cabida a otros profesionales en nuestros centros para hacer lo que no sepamos. Como ejemplo indicativo de la necesidad de replantearnos como profesión, en Malmö buscaron personal distinto para su nueva biblioteca, ante la conciencia de que las relaciones personales resultaban clave para el éxito del nuevo proyecto de servicios. No se contrataron bibliotecarios porque "no podían ver las posibilidades de esta nueva concepción [de biblioteca]" (Berndtson, 2012).

\section{Servicios para, con o sin el usuario}

Son los usuarios los que finalmente decidirán el destino de las bibliotecas (Choy, 2010, p. 62): si no les interesan nuestros servicios, si no nos usan, estamos condenados a la desaparición. El servicio está así totalmente vinculado con el usuario, con sus necesidades. Por eso se deben crear propuestas que les interesen, que les sirvan, y para ello hay que contar con ellos. Los servicios se tienen que prestar en función de los usuarios, no de nuestras funciones y organización. Hay que tomar su punto de vista, teniendo en cuenta que los servicios son cada vez menos transaccionales y más relacionales (Berndtson, 2012). La biblioteca como máquina prestadora de servicios, se orienta hacia el usuario y sus necesidades. Como dice Domínguez-Sanjurjo (1999, p. 54), "si no están orientadas al usuario ¿̇a quién están orientadas?".

Hay que implementar servicios para los usuarios, pero si queremos que tengan éxito, nos debemos acostumbrar a diseñarlos con ellos. Se acabó el despotismo ilustrado según el cual se ofrecían servicios no demandados porque eran de gran calidad, pues puede que no le sirvan ni interesen a nadie. Hasta hace no mucho los bibliotecarios se sentían culpables si diseñaban un servicio en función de los deseos de los usuarios. Como profesionales debían saber lo que los usuarios verdaderamente necesitaban. Se demostraba una actitud paternalista (Esson et al., 2012), por la que se decidía por los usuarios, que no siempre sabían lo que necesitaban.

\section{Los servicios están totalmente vincula- dos con los usuarios: si no les interesan nuestros servicios, si no nos usan, esta- mos condenados a la desaparición}

Esto no significa que eliminemos nuestra capacidad innovadora e imaginativa. No sólo se deben crear servicios a remolque de las necesidades, sino que habrá que adelantarse en lo posible a las mismas: "bibliotecas que buscan y consiguen la sorpresa entre sus usuarios" (García-Gómez, 2013). Lo importante es lo que necesita y quiere el usuario, no lo que nosotros pensamos que necesita.

Tampoco significa que la biblioteca adopte sin más cualquier servicio que pueda tener aceptación. Bastante nos calificaron en su momento como videoclubs, ante la incomprensión del préstamo de audiovisuales. La biblioteca orientada a la demanda tiene unos límites éticos marcados por su misión (Lozano-Díaz, 2003).

Además, para que los servicios tengan éxito deben refinarse con la retroalimentación proporcionada por los usuarios. Deben estar bajo una evaluación constante y modificarse en función de los resultados (Esson et al., 2012).

El Manifiesto IFLA/Unesco de 1994 se vuelca en dos aspectos, accesibilidad y alcance:

- que los servicios de la biblioteca se adapten a las necesidades de la comunidad en su conjunto, y

- que lleguen a todas partes, con presencia más allá de sus paredes (Gallo-León, 2008).

Si el objetivo de un centro bibliotecario es satisfacer a tantos usuarios como sea posible, la función primordial de las actividades es ofrecer los servicios que necesitan la mayor variedad posible de usuarios.

En esto las tecnologías ayudan a ampliar nuestro rango de usuarios. Facilitan que lleguemos hasta ellos, pero también extender nuestra oferta y hacerla más específica. Esto puede ser especialmente interesante por el fenómeno del longtail $^{9}$, según el cual hay unos pocos servicios/productos que interesan a un grupo muy grande de personas, pero hay otros muchos que interesan a pocas personas, a nichos de usuarios. Esto se plasma en una gráfica con un punto álgido (los productos de gran difusión o mainstream), bajando rápidamente hacia los productos que interesan a muchos grupos minoritarios diferentes, formando así una larga cola. Si somos capaces de ofertar servicios de interés para muchos de estos grupos minoritarios, la suma será similar a los 
de los productos estrella, captando un gran número final de personas (Casey; Savastinuk, 2006). Internet ha permitido que grupos con intereses más o menos minoritarios accedan fácilmente a productos que antes ni conocían. Desde las bibliotecas se puede utilizar la misma táctica, sobre todo si se apoya en un trabajo distribuido y cooperativo. El fenómeno también tiene un reverso: la difícil supervivencia de bibliotecas sucursales y puntos de lectura, cuya oferta de servicios es muy limitada.

\section{Los servicios son los que hacen que el espacio agradable que queremos con- seguir sea el lugar al que apetezca y se tenga que ir}

De la misma forma, se debe tener en cuenta la aplicación bibliotecaria de las tecnologías de la participación. Con ellas, la biblioteca puede mejorar su interactividad con los usuarios, estableciendo una relación plenamente abierta (Merlo-Vega, 2007), en la que el usuario es un colaborador de la misma. Así, se puede cumplir con la máxima según la cual, si se quiere incrementar el atractivo y el valor de la biblioteca de cara a los usuarios, se debe estudiar la implementación de servicios participativos y personalizados (Casey; Savastinuk, 2006). La tecnología ayuda a llegar a personas que físicamente son inaccesibles o que no están interesadas en nuestros servicios; y de una manera eficaz y eficiente: rápida, barata, sencilla y muy difundida. Estas tecnologías nos permiten cubrir un buen trozo de la larga cola de usuarios nicho.

Es el volumen de uso de nuestros servicios lo que determinará nuestra supervivencia

De cualquier forma, ya no basta con la calidad y valor intrínseco del servicio (Choy, 2010). Para ser elegido por los usuarios también debe ser conveniente, su uso debe ser ventajoso sobre otras alternativas en términos de sencillez, usabilidad, comodidad, esfuerzo o tiempo invertido. Además los servicios deben llamar la atención, ser atractivos para destacar entre la inmensa oferta externa. Esto no será suficiente, pues también debemos conseguir que conozcan nuestra oferta. Cualquier bibliotecario sabe de la sorpresa de muchos usuarios ante la variedad de la oferta de su centro cuando la descubre. Para todo ello echaremos mano de múltiples mecanismos de comunicación y promoción, siendo importantísimo reforzar estas actividades. Al respecto, como señala González-Fernández-Villavicencio (2009), resulta destacable que las bibliotecas se ocupen de realizar "esmerados" planes estratégicos, sin preocuparse de implantar planes de marketing.

Se ha publicado mucho sobre la promoción de los servicios, sobre todo usando métodos tecnológicos: redes sociales, movilidad, QR, etc. Las tendencias ${ }^{10}$ marcan que nos debemos acercar al usuario con rasgos humanos, cálidos, cerca-

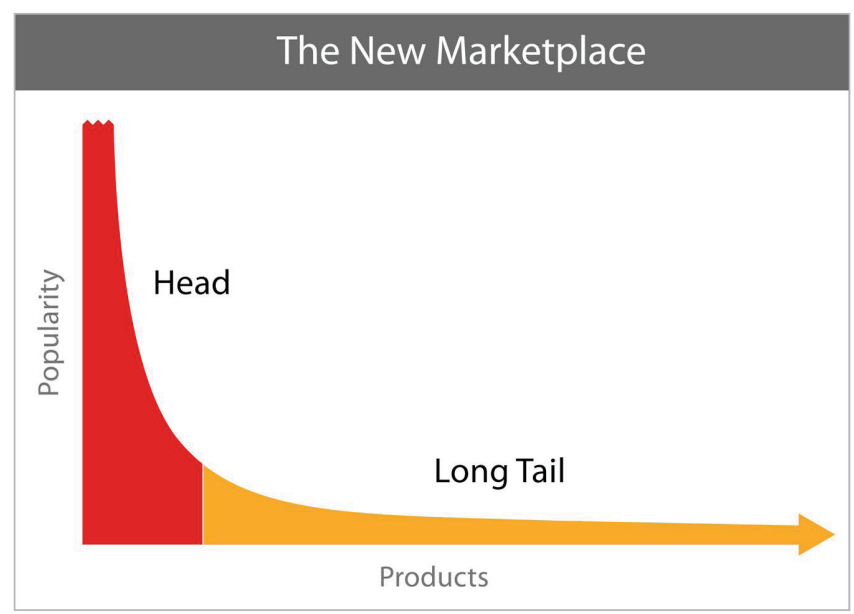

Figura 3. Gráfico explicativo de la long tail, tomado del blog de Chris Anderson, autor de este concepto.

http://www.longtail.com/about.html

nos; y poniendo en comunicación a los usuarios entre ellos (otra vez el uso de las redes). Sin embargo resulta complicado llamar la atención de forma efectiva sobre nuestros servicios. Para ello contamos con herramientas como el diseño atractivo del entorno físico y digital. Pero además podemos estar con el usuario. La biblioteca debe estar presente en los entornos de trabajo de nuestros usuarios, bien físicamente (bibliotecarios incrustados), bien virtualmente (por ejemplo con la presencia en campus virtuales con banners, servicios de referencia online, etc.)

Igualmente, existe abundante bibliografía sobre los métodos para conocer las necesidades y opiniones de los usua$\operatorname{rios}^{11}$, en especial con estudios y encuestas, pues han sido bastante populares en los países anglosajones. Además se puede explotar la interpretación de los indicadores de calidad, pero lo habitual son encuestas, grupos focales o entrevistas individuales, entre otros (Esson et al., 2012). Como alternativa más próxima, aunque con las limitaciones que la privacidad nos impone, se puede usar el data mining y otras herramientas de explotación de big data para acercarnos al usuario, segmentar la oferta y proponer servicios que le puedan interesar ${ }^{12}$.

La función primordial de todas las actividades de la biblioteca es ofrecer los servicios que necesita la mayor variedad posible de usuarios

\section{Conclusiones}

En definitiva los servicios bibliotecarios se encuentran en el mismo cambio de época que la biblioteca misma, pues son su fundamento y razón de ser. Es por ello que la renovación de estos servicios debe ser el camino para un nuevo impulso de las bibliotecas. En el proceso puede que incluso pierdan el nombre, pero desde luego deberán cambiar la idea que de ellas tiene la sociedad. Esta evolución será imposible si no se diseñan de acuerdo con las preferencias y necesidades de todos los usuarios (también de los potenciales), y con ellos participando en el desarrollo. 


\section{Notas}

1. Traducción propia de "there has never been a more exciting time to be in libraries: it is both daunting and empowering", frase de Lynne Brindley en el prólogo de McKnight (2010), citado por Stevenson (2011).

2. El origen de este servicio central de las bibliotecas durante siglo y medio se sitúa en 1876, cuando Samuel-Swett Green publica su célebre "Personal relations between librarians and readers" en Library journal.

\section{3. http://www.ala.org/transforminglibraries/future}

Se puede consultar la interesante recopilación de artículos recientes sobre el tema realizada por Anglada (2014, p. $603)$, quien parece haber pasado del optimismo al pesimismo (tal vez sólo como vía de agitación) en el artículo citado.

4. Para ello está este número de EPI y otros artículos, algunos de los cuales se pueden encontrar en la bibliografía. También puede resultar interesante la consulta del estudio Prospectiva 2020 (Gallo-León, 2013), sobre todo el apartado 9.

\section{Citando a: De-Rosa; Cantrell (2005); Gauder (2010).}

6. Esperemos que este esfuerzo no quede como en el humorístico vaticinio sobre las herramientas de descubrimiento y la muerte de las bibliotecas que recogía el Grupo Durga (2013) en la traducción de un post de Aaron Tay (2013).

7. El término "tercer lugar" fue acuñado por el sociólogo Ray Oldenburg en su libro The great good place (1989). Se entiende como un espacio complementario dedicado a la vida social de la comunidad y se refiere a las zonas donde la gente puede encontrarse, reunirse e interrelacionarse de manera informal. Espacios que no sean ni nuestra casa (primer lugar) ni la oficina (segundo lugar).

http://bibliotecas2029.com/2012/05/23/tercer-lugar

8. No siempre tenemos tan controlada como creemos la privacidad de nuestros usuarios. Sobre el tema, resulta de gran interés el post "Privacidades" de Grupo Durga (2014). https://bibliotecas2029.wordpress.com/2014/10/28/ privacidades

9. El término fue acuñado por Chris Anderson (2014), editor jefe de Wired, en un conocido artículo que revolucionó el mundo de la gestión empresarial. La información se actualiza en el blog The long tail. Además hay el artículo específico sobre bibliotecas y long tail de Mossman (2006).

http://www.longtail.com

10. Véase New Jersey State Library (2014).

http://www.njstatelib.org/services_for_libraries/consulting_ services/library_trustees

11. Como ejemplo modélico de acercamiento a los usuarios para recabar sus opiniones, el de la biblioteca de Helsinki (Miettinen, 2013), para la construcción de su nuevo edificio.

12. Para conocer más sobre el estado de la cuestión de los big data y de sus aplicaciones, véase Serrano-Cobos (2014); y sobre ejemplos prácticos de su uso para mejora de servicios, Kovačević; Devedžić; Pocajt (2010).

\section{Bibliografía}

Anderson, Chris (2004). "The long tail". Wired, October, n. 12.10.

http://archive.wired.com/wired/archive/12.10/tail.html

Anglada, Lluís (2012). "Bibliotecas universitarias: cabalgando la tecnología, siguiendo al usuario". El profesional de la información, v. 21, n. 6, noviembre-diciembre, pp. 553-556. http://www.elprofesionaldelainformacion.com/ contenidos/2012/noviembre/01.pdf http://dx.doi.org/10.3145/epi.2012.nov.01

Anglada, Lluís (2014). "Are libraries sustainable in a world of free, networked, digital information?". El profesional de la información, v. 23, n. 6, november-december, pp. 603- 611. http://dx.doi.org/10.3145/epi.2014.nov.07

Berndtson, Maija (2012). "What and why libraries?: looking at what libraries might look like and why we still need them now and into the future". Library hi tech news, v. 29, n. 4, pp. 13-15.

http://www.emeraldinsight.com/doi/full/10.1108/07419051211249464 http://dx.doi.org/10.1108/07419051211249464

Bolt, Nancy (2014). Libraries from now on: Imagining the future of libraries. ALA summit on the future of libraries. Report to ALA membership. Chicago: American Library Association.

http://goo.gl/OW6wfU

Casey, Michael E.; Savastinuk, Laura C. (2006). "Library 2.0 service for the next-generation library". Library journal, Sept., v. 131, n. 14, p. 40.

http://lj.libraryjournal.com/2010/05/technology/ library-2-0/\#

Choy, Fatt-Cheong (2011). "From library stacks to libraryin-a-pocket: will users be around?". Library management, v. 32, n. $1 / 2$, pp. 62-72.

http://dx.doi.org/10.1108/01435121111102584

Coffman, Steve (2012). "The decline and fall of the library empire". Searcher, v. 20, n. 3.

http://www.infotoday.com/searcher/apr12/Coffman--TheDecline-and-Fall-of-the-Library-Empire.shtml

De-Rosa, Cathy; Cantrell, Joanne et al. (2005). Perceptions of libraries and information resources. A report to the OCLC membership, $290 \mathrm{pp}$.

http://oclc.org/reports/2005perceptions.en.html

Domínguez-Sanjurjo, Ramona (1999). “La biblioteca al servicio del usuario". Educación y biblioteca, v. 11, n. 100, pp. 54-56.

http://hdl.handle.net/10366/115385

Esson, Rachel; Stevenson, Alison; Gildea, Maureen; Roberts, Sue (2012). "Library services for the future: engaging with our customers to determine wants and needs". Library management, v. 33, n. 8/9, pp. 469-478.

http://dx.doi.org/10.1108/01435121211279830

Gallo-León, José-Pablo (2008). “Innovación en los servicios de bibliotecas públicas orientadas al usuario. El caso de la 
Biblioteca Regional de Murcia". El profesional de la información, marzo-abril, v. 17, n. 2, pp. 144-154.

http://www.elprofesionaldelainformacion.com/ contenidos/2008/marzo/03.pdf

http://dx.doi.org/10.3145/epi.2008.mar.03

Gallo-León, José-Pablo (coord.) (2013). Prospectiva 2020: Las diez áreas que más van a cambiar en nuestras bibliotecas en los próximos años. Consejo de Cooperación Bibliotecaria, Ministerio de Cultura.

http://www.mcu.es/bibliotecas/docs/MC/ConsejoCb/ GruposTrabajo/GE_prospectiva/Estudioprospectiva2020.pdf

García-Gómez, F. Javier (2013). "Innovación en la biblioteca pública española: dónde estamos y hacia dónde vamos". Revista general de información y documentación, v. 23, n.1, pp. 133-150.

http://dx.doi.org/10.5209/rev_RGID.2013.v23.n1.41449

Gauder, Brad (ed.) (2010). Perceptions of libraries: context and community. A report to the OCLC membership. Dublin, Ohio: OCLC. ISBN: 9781556533952

http://www.oclc.org/reports/2010perceptions/2010percept ions_all_singlepage.pdf

González-Fernández-Villavicencio, Nieves (2009). “Bibliotecas y marketing en red". BiD: textos universitaris de biblioteconomia i documentació, desembre, n. 23.

http://www.ub.edu/bid/23/gonzalez2.htm

http://dx.doi.org/10.1344/105.000001479

Grupo Durga (2012). "Cómo ir perdiendo usuarios y servicios por el camino hacia la biblioteca del futuro". Bibliotecas 2029: Documentos y debates sobre el futuro de las bibliotecas, 27 octubre.

https://bibliotecas2029.wordpress.com/2012/10/27/comoir-prdiendo-usuarios

Grupo Durga (2013). "La encontraron muerta". Bibliotecas 2029: Documentos y debates sobre el futuro de las bibliotecas, 11 noviembre.

https://bibliotecas2029.wordpress.com/2013/11/11/ discovery-died

Grupo Durga (2014). "Privacidades". Bibliotecas 2029: Documentos y debates sobre el futuro de las bibliotecas, 28 octubre. https://bibliotecas2029.wordpress.com/2014/10/28/ privacidades

Kovačević, Ana; Devedžić, Vladan; Pocajt, Viktor (2010). "Using data mining to improve digital library services". The electronic library, v. 28, n. 6, pp. 829-843.

http://dx.doi.org/10.1108/02640471011093525

Lozano-Díaz, Roser (2003). “Lectores, usuarios, clientes: perspectiva de una biblioteca pública al servicio de los ciudadanos". En: I Jornadas del Sistema Nacional de Bibliotecas de Euskadi, 2003.

http://www.euskadi.net/r33-2288/es/contenidos/informacion/ jornadas_bibliotecarias/es_8126/adjuntos/jorna02.pdf

Lozano-Díaz, Roser (2008). "Innovación en bibliotecas públicas: algo nuevo, algo útil, algo de calidad". El profesional de la información, marzo-abril, v. 17, n. 2, pp. 129-134.

http://www.elprofesionaldelainformacion.com/ contenidos/2008/marzo/01.pdf

http://dx.doi.org/10.3145/epi.2008.mar.01

McKnight, Sue (ed.) (2010). Envisioning future academic library services: initiatives, ideas and challenges. London: Facet Publishing. ISBN: 9781856046916.

Merlo-Vega, José-Antonio (2007). "Las tecnologías de la participación en la biblioteca". Educación y biblioteca, v. 19, n. 161 , pp. 63-68 http://eprints.rclis.org/handle/10760/10558

Miettinen, Virve (2013). "The future library designed with you". Scandinavian library quarterly, v. 46, n. 2.

http://slq.nu/?article=volume-46-no-2-2013-6

Mossman, Katherine (2006). "Serving the niche: viewing libraries through Chris Anderson's 'long tail' lens". Library journal, July, v. 131, n. 12, pp. 38-40.

http://lj.libraryjournal.com/2006/07/ljarchives/servingthe-niche

New Jersey State Library (2014). “5 public library marketing trends to expect in 2015". NJSL marketing Blog, December 19. http://www.njstatelib.org/blog/marketing/2014/5-publiclibrary-marketing-trends-expect-2015

Serrano-Cobos, Jorge (2014). "Big data y analítica web. Estudiar las corrientes y pescar en un océano de datos". El profesional de la información, v. 23, n. 6, noviembre-diciembre, pp. 561-565.

http://www.elprofesionaldelainformacion.com/ contenidos/2014/nov/01.pdf

http://dx.doi.org/10.3145/epi.2014.nov.01

Stevenson, Valerie (2011). "Envisioning future academic library services: initiatives, ideas and challenges. Edited by Sue McKnight" [Review]. Sconul focus, n. 51, pp. 80-81. http://www.sconul.ac.uk/sites/default/files/ documents/25_0.pdf

Tay, Aaron (2013). "The day library discovery died - 2035". http://musingsaboutlibrarianship.blogspot.com. es/2013/09/the-day-library-discovery-died-2035.html

Walker, Scott (2011). "Distinctive signifiers of excellence: library services and the future of the academic library". College \& research libraries, January, v. 72, n. 1, pp. 6-8. http://dx.doi.org/10.5860/0720006 


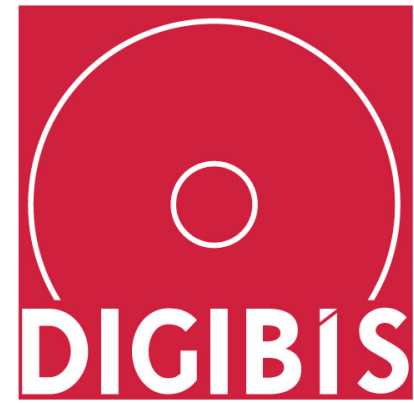

Software de gestión para Bibliotecas, Archivos y Museos

Digitalización enriquecida de fondo antiguo y patrimonial

Transformando

y enriqueciendo la biblioteca con aplicaciones de gestión digital de nueva generación.

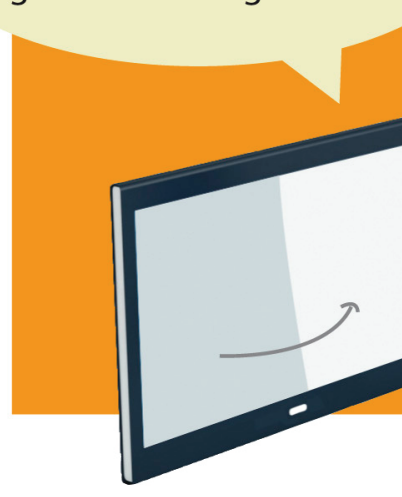

Facilitando el acceso a recursos desde cualquier lugar, en cualquier momento, con cualquier dispositivo.
Siendo recolectada automáticamente por Hispana, Europeana y otros agregadores internacionales.

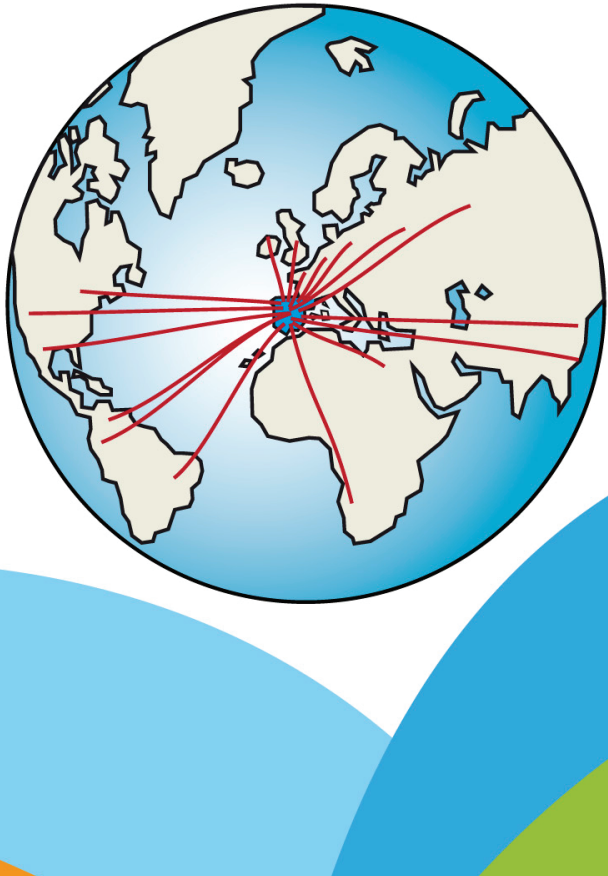
er

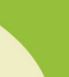

